CARDIOVASCULAR MEDICINE

\title{
Pulsed wave tissue Doppler imaging for the quantification of contractile reserve in stunned, hibernating, and scarred myocardium
}

\author{
M Bountioukos, A F L Schinkel, J J Bax, V Rizzello, R Valkema, B J Krenning, E Biagini, \\ E C Vourvouri, J R T C Roelandt, D Poldermans
}

Heart 2004;90:506-510. doi: 10.1136/hrt.2003.018531

See end of article for authors' affiliations

......................

Correspondence to:

Dr Don Poldermans,

Thoraxcenter Room $\mathrm{Ba}$

300, Department of

Cardiology, Erasmus

Medical Centre, $\mathrm{Dr}$

Molewaterplein 40, 3015

GD Rotterdam,

Netherlands:

d.poldermans@

erasmusmc.nl

Accepted

8 September 2003

\begin{abstract}
Objectives: To assess whether quantification of myocardial systolic velocities by pulsed wave tissue Doppler imaging can differentiate between stunned, hibernating, and scarred myocardium.

Design: Observational study.

Setting: Tertiary referral centre.

Patients: 70 patients with reduced left ventricular function caused by chronic coronary artery disease. Methods: Pulsed wave tissue Doppler imaging was done close to the mitral annulus at rest and during low dose dobutamine; systolic ejection velocity (Vs) and the difference in $\mathrm{V}_{s}$ between low dose dobutamine and the resting value $\left(\Delta \mathrm{V}_{\mathrm{s}}\right)$ were assessed using a six segment model. Assessment of perfusion (with Tc-99mtetrofosmin SPECT) and glucose utilisation (by ${ }^{18} \mathrm{~F}$-fluorodeoxyglucose SPECT) was used to classify dysfunctional regions (by resting cross sectional echocardiography) as stunned, hibernating, or scarred. Results: 253 of 420 regions $(60 \%)$ were dysfunctional. Of these, $132(52 \%)$ were classified as stunned, 25 $(10 \%)$ as hibernating, and $96(38 \%)$ as scarred. At rest, Vs in stunned, hibernating, and scar tissue was, respectively, $6.3(1.8), 6.6(2.2)$, and $5.5(1.5) \mathrm{cm} / \mathrm{s}(\mathrm{p}=0.001$ by ANOVA). There was a gradual decline in $V_{s}$ during low dose dobutamine infusion between stunned, hibernating, and scar tissue $(8.3$ (2.6) $\vee 7.8(1.5) \vee 6.8(1.9) \mathrm{cm} / \mathrm{s}, \mathrm{p}<0.001$ by ANOVA). $\Delta$ Vs was higher in stunned $(2.1(1.9) \mathrm{cm} / \mathrm{s})$ than in hibernating $(1.2(1.4) \mathrm{cm} / \mathrm{s}, \mathrm{p}<0.05)$ or scarred regions $(1.3(1.2) \mathrm{cm} / \mathrm{s}, \mathrm{p}=0.001)$.

Conclusions: Quantitative tissue Doppler imaging showed a gradual reduction in regional velocities between stunned, hibernating, and scarred myocardium. Dobutamine induced contractile reserve was higher in stunned regions than in hibernating and scarred myocardium, reflecting different severities of myocardial damage.
\end{abstract}

l一․ patients with chronic coronary artery disease, it is important to differentiate between stunned, hibernating, and scarred myocardial tissue. Patients with substantial viability (stunned or hibernating myocardium) may be candidates for coronary revascularisation, whereas patients with irreversibly damaged myocardium will probably not benefit from revascularisation. ${ }^{1-3}$ The development of stress echocardiography and nuclear imaging techniques has enabled us to differentiate viable from non-viable myocardium. $^{4-8}$ Moreover, the combined assessment of perfusion and glucose utilisation permits the differentiation of normally perfused dysfunctional myocardium (stunned myocardium) from chronically hypoperfused dysfunctional myocardium (hibernating myocardium). ${ }^{9-11}$ Although it is likely that stunned and hibernating myocardium do not represent different entities but consist of a continuum of myocardial dysfunction in ascending order of severity, it is clinically relevant to delineate stunning from hibernation. Preliminary data show that hibernating myocardium may need a longer time to recover contractile function fully than stunned tissue. ${ }^{12}$ Our aim in this study was to quantify, using pulsed wave tissue Doppler imaging, the differences in regional systolic wall motion and contractile reserve in stunned, hibernating, and scarred myocardial tissue.

\section{METHODS}

Patient population

Seventy patients with chronic coronary artery disease (previous myocardial infarction or angiographically proven coronary artery disease) were studied at least six months after any previous myocardial infarct. Patients with idiopathic dilated cardiomyopathy, significant valvar heart disease, or a suboptimal acoustic window were not included in the study.

The study protocol was as follows. First, left ventricular ejection fraction was assessed by radionuclide ventriculography. Next, regional contractile function was evaluated by resting cross sectional echocardiography. Then low dose dobutamine ( 5 and $10 \mu \mathrm{g} / \mathrm{kg} / \mathrm{min}$ ) was infused and contractile reserve was assessed. The resting regional wall motion systolic velocities (Vs) and change in velocities during dobutamine infusion (contractile reserve, $\Delta \mathrm{Vs}$ ) were then quantified by pulsed wave tissue Doppler imaging. Finally, single photon emission computed tomography (SPECT) imaging was done, and perfusion and glucose utilisation were evaluated using ${ }^{99 \mathrm{~m}}$ Tc-tetrofosmin and ${ }^{18} \mathrm{~F}$-fluorodeoxyglucose $\left({ }^{18} \mathrm{~F}-\mathrm{FDG}\right)$, respectively. According to the perfusionmetabolism patterns on SPECT imaging, dysfunctional myocardium was categorised as stunned, hibernating, or scar tissue. Quantitative tissue Doppler imaging data were related to the SPECT findings.

\footnotetext{
Abbreviations: ANOVA, analysis of variance; ${ }^{18} \mathrm{~F}-\mathrm{FDG},{ }^{18} \mathrm{~F}$ fluorodeoxyglucose; NYHA, New York Heart Association; SPECT, single photon emission computed tomography; $V_{s}$, systolic velocity; $\Delta \mathrm{V}_{\mathrm{s}}$, difference in systolic velocity between rest and during low dose dobutamine infusion
} 
All patients gave their informed consent, and the local ethics committee approved the study protocol.

\section{Radionuclide ventriculography to assess left ventricular ejection fraction}

After injection of ${ }^{99 \mathrm{~m}} \mathrm{Tc}(740 \mathrm{MBq})$, radionuclide ventriculography was done at rest with the patient in the supine position. A small field of view gamma camera (Orbiter; Siemens, Erlangen, Germany) was used, oriented in a $45^{\circ} \mathrm{left}$ anterior oblique position with a $5-10^{\circ}$ caudal tilt. The left ventricular ejection fraction was calculated using standard methods. ${ }^{13}$

\section{Cross sectional echocardiography to assess resting contractile function and contractile reserve}

We used a commercially available imaging system (Sonos 5500; Hewlett Packard, Andover, Massachusetts, USA) and a 1.8 $\mathrm{MHz}$ transducer employing second harmonic imaging to optimise visualisation of the endocardial border. ${ }^{14}$ Cross sectional imaging was done with the patient in the left lateral position. Standard views were recorded onto an optical disk (cine loop format). For the assessment of contractile reserve in dysfunctional myocardium, we used dobutamine stress echocardiography, as described previously. ${ }^{15}$ After the resting echocardiographic study, dobutamine was given intravenously at doses of 5 and $10 \mu \mathrm{g} / \mathrm{kg} / \mathrm{min}$ for five minutes. Two experienced observers, unaware of the clinical data or the SPECT results, scored the digitised echocardiograms off-line. In cases of disagreement, a third observer reached a majority decision. Six regions were evaluated, including lateral, inferior, infero-septal, anteroseptal, anterior, and posterior. ${ }^{16}$ Regional wall motion and systolic wall thickening were scored using a five point grading scale: 1, normal; 2, mildly hypokinetic; 3 , severely hypokinetic; 4, akinetic; and 5, dyskinetic. Regions with severe hypokinesia, akinesia, or dyskinesia were considered abnormal; segments with mild hypokinesia were considered normal.

Tissue Doppler imaging to quantify resting contractile function and contractile reserve

The same six segment model was used for pulsed wave tissue Doppler imaging. A pulse repetition frequency of $45-60 \mathrm{kHz}$ and a sample volume of $4 \mathrm{~mm}^{3}$ were used. The measurement of myocardial velocity was sampled in three apical views (four chamber, two chamber, and three chamber) close to the mitral annulus and during a minimum of five consecutive beats, in order to minimise the variability induced by respiration. The depth of the sample volume of every region was kept constant during dobutamine stress echocardiography to ensure that left ventricular myocardium was sampled close to the mitral annulus. The Doppler velocity profiles and ECG tracings were simultaneously stored on optical disk. All measurements were done off-line using a computer assisted drawing system. The velocity values $(\mathrm{cm} / \mathrm{s})$ were obtained on calibrated still frames by manually measuring the distance between the zero baselines and the peak Doppler profile of the ejection phase, in reference to the ECG. Cardiac cycles with extrasystolic beats, post-extrasystolic beats, or rhythm disturbance were excluded. Recordings and measurements were done at baseline and during low dose $(10 \mu \mathrm{g} / \mathrm{kg} / \mathrm{min})$ dobutamine infusion.

\section{SPECT tissue characterisation: assessment of stunning, hibernation, and scar}

All patients underwent dual isotope simultaneous acquisition SPECT. Resting ${ }^{99 \mathrm{~m}}$ Tc-tetrofosmin SPECT (600 MBq) was used to assess regional perfusion. Myocardial glucose utilisation was evaluated by ${ }^{18}$ F-FDG SPECT (185 MBq). To optimise cardiac ${ }^{18} \mathrm{~F}-\mathrm{FDG}, 500 \mathrm{mg}$ of Acipimox (Byk, Netherlands) was given orally to all patients. ${ }^{17} \mathrm{~A}$ triple head gamma camera (Prism 3000XP; Picker, Cleveland, Ohio, USA) was used. The camera was equipped with commercially available high energy $511 \mathrm{keV}$ collimators. ${ }^{18}$ The energies were centred on the $140 \mathrm{keV}$ photon peak of ${ }^{99 \mathrm{~m}} \mathrm{Tc}$ tetrofosmin with a $15 \%$ window, and on the $511 \mathrm{keV}$ photon peak of ${ }^{18}$ F-FDG with a $15 \%$ window. Data were acquired over $360^{\circ}$ ( 120 sectors of $\left.3^{\circ}\right)$, and total imaging time was 32 minutes. The data were stored in a $64 \times 64,16$ bit matrix. The images were reconstructed by filtered back projection using a Butterworth filter (cut off frequency, 0.17 cycles per pixel); $6 \mathrm{~mm}$ thick (one pixel) transaxial slices were obtained. Subsequently, standard short and long axis projections perpendicular to the heart axis were reconstructed. The same six regions as for the echocardiographic analysis were used.

Both ${ }^{99 m}$ Tc-tetrofosmin and ${ }^{18}$ F-FDG studies were analysed quantitatively (segments normalised to maximum tracer uptake). Dysfunctional segments (identified by resting echocardiography) were evaluated for perfusion and glucose utilisation. ${ }^{4}{ }^{20}$ Dysfunctional segments with normal perfusion (normalised ${ }^{99 \mathrm{~m}} \mathrm{Tc}$-tetrofosmin uptake of $>80 \%$ ) were classified as stunned. Dysfunctional segments with a perfusion defect (normalised ${ }^{99 \mathrm{~m}}$ Tc-tetrofosmin uptake $<80 \%$ ) were classified as hibernating when a perfusion- $-{ }^{18}$ F-FDG mismatch was present (relative increase of ${ }^{18} \mathrm{~F}$-FDG uptake $\geqslant 10 \%$, compared with ${ }^{99 m}$ Tc-tetrofosmin uptake). Dysfunctional segments with a perfusion defect were classified as scar tissue when a perfusion- ${ }^{18}$ F-FDG match was present $\left(<10 \%\right.$ difference in tracer activities). ${ }^{4}{ }^{19} 20$

\section{Statistical analysis}

Dichotomous variables are presented as $\mathrm{n}(\%)$ and continuous variables as mean (SD). Comparisons between severely and non-severely dysfunctional regions were made using the Student $t$ test. Comparisons between normal, mildly hypokinetic, severely hypokinetic, and akinetic/dyskinetic regions, as well as between stunned, hibernating, and scarred regions, were made by one way analysis of variance (ANOVA). Bonferroni analysis was used to assess significance during multiple comparisons. Significance of all statistical tests was assumed at the 0.05 probability level.

\section{RESULTS}

\section{Patient characteristics}

The baseline clinical characteristics of the 70 patients are summarised in table 1. Mean left ventricular ejection fraction was $31(10) \%$ (range 10-45\%), and the New York Heart Association (NYHA) functional class was on average 3.0 (1.2). The majority of patients (53 patients, $76 \%$ ) were in NYHA class III or IV.

\section{Cross sectional echocardiography to assess resting contractile function and contractile reserve}

Resting echocardiography was undertaken in 420 regions. Of these, $167(40 \%)$ had normal contractile function (normokinesia), while 253 (60\%) were dysfunctional, including 164 severely hypokinetic and 89 akinetic/dyskinetic regions. Of the 253 dysfunctional regions, 181 (72\%) showed contractile reserve during dobutamine infusion (increase in wall motion score index by one grade or more during dobutamine infusion). The haemodynamic response during low dose dobutamine infusion is shown in table 2. The administration of dobutamine was well tolerated by all patients. The protocol was completed in all patients without serious side effects. 
Table 1 Clinical baseline characteristics of the 70 study patients

\begin{tabular}{ll}
\hline Male sex & $59(84 \%)$ \\
Age (years) & $62(10)$ \\
New York Heart Association functional class & $3.0(1.2)$ \\
Left ventricular ejection fraction (\%) & $31(10)$ \\
Previous myocardial infarction & $62(89 \%)$ \\
Previous coronary artery bypass graft surgery & $15(21 \%)$ \\
Previous percutaneous transluminal coronary angioplasty & $20(29 \%)$ \\
Hypertension* & $17(24 \%)$ \\
Diabetes mellitust & $8(11 \%)$ \\
Hypercholesterolaemiał & $27(39 \%)$ \\
Current smoking & $15(21 \%)$ \\
Angina pectoris & $40(57 \%)$ \\
Drug treatment & \\
Aspirin/oral anticoagulants & $62(89 \%)$ \\
Angiotensin converting enzyme inhibitors & $58(83 \%)$ \\
Diuretics & $35(50 \%)$ \\
$\beta$ Blockers & $42(60 \%)$ \\
Digoxin & $12(17 \%)$ \\
\hline
\end{tabular}

Data are $\mathrm{n}(\%)$ or mean (SD).

*Defined as blood pressure $\geqslant 140 / 90 \mathrm{~mm} \mathrm{Hg}$, or treatment with antihypertensive drugs.

tPatients receiving oral antidiabetics or insulin.

$\neq$ Defined as a total cholesterol $\geqslant 6.4 \mathrm{mmol} / /$ or treatment with lipid lowering drugs.

Tissue Doppler imaging to quantify resting contractile function and contractile reserve

Myocardial systolic velocity of normal or mildly hypokinetic regions (non-dysfunctional regions) was $6.8(2.0) \mathrm{cm} / \mathrm{s}$ at rest and $9.2(3.3) \mathrm{cm} / \mathrm{s}$ during low dose dobutamine challenge, while $\Delta V \mathrm{~V}$ was $2.4(2.8) \mathrm{cm} / \mathrm{s}$. Dysfunctional regions had significantly lower velocities: $6.2(1.6) \mathrm{cm} / \mathrm{s}$ at rest $(\mathrm{p}<0.001)$ and $7.6(2.0) \mathrm{cm} / \mathrm{s}$ at low dose dobutamine $(\mathrm{p}<0.001)$, with $\Delta$ Vs reaching $1.4(1.5) \mathrm{cm} / \mathrm{s}(\mathrm{p}<0.001)$. Figure 1 illustrates the systolic velocities at rest and during low dose dobutamine infusion, and $\Delta$ Vs in normal, mildly hypokinetic, severely hypokinetic, and akinetic/dyskinetic regions, according to the cross sectional echocardiographic assessment. Comparison between the four groups (by ANOVA) showed significant differences in Vs at rest $(p=0.003)$ and during low dose dobutamine infusion $(\mathrm{p}<0.001)$, as well as in $\Delta$ Vs $(\mathrm{p}<0.001)$.

In dysfunctional regions with contractile reserve during low dose dobutamine challenge, maximum Vs was 6.1 (1.6) $\mathrm{cm} / \mathrm{s}$ at rest and $8.0(2.2) \mathrm{cm} / \mathrm{s}$ during low dose dobutamine, and $\Delta$ Vs was $1.9(2.1) \mathrm{cm} / \mathrm{s}$. In dysfunctional regions without contractile reserve, Vs was $6.2(2.0) \mathrm{cm} / \mathrm{s}$ at rest $(\mathrm{p}=0.41)$ and $7.3(2.2) \mathrm{cm} / \mathrm{s}$ during low dose dobutamine $(\mathrm{p}=0.04)$, and $\Delta V \mathrm{~V}$ was $1.1 \quad(2.0) \mathrm{cm} / \mathrm{s}$ $(\mathrm{p}=0.008)$.

\section{SPECT imaging, tissue characterisation}

Dual isotope SPECT imaging showed that 132 of 253 dysfunctional regions (52\%) had normal perfusion and glucose utilisation; these segments were considered stunned. Reduced perfusion and relatively preserved glucose utilisation (hibernating myocardium) was observed in 25 of the dysfunctional regions (10\%). The remaining regions (96; $38 \%$ ) had concordantly reduced perfusion and metabolism and were considered to be scar tissue.

\section{Tissue Doppler imaging in stunned, hibernating, and scarred regions \\ Vs at rest}

In the dysfunctional regions, Vs at rest in stunned, hibernating, and scar tissue was, respectively, 6.3 (1.8), 6.6 (2.2), and $5.5(1.5) \mathrm{cm} / \mathrm{s}(\mathrm{p}=0.001$ by ANOVA).

\section{Vs during low dose dobutamine}

During low dose dobutamine infusion there was a gradual decline in Vs in stunned, hibernating, and scar tissue $(8.3$ (2.6) cm/s $\vee 7.8(1.5) \mathrm{cm} / \mathrm{s} \vee 6.8$ (1.9) cm/s; p < 0.001 by ANOVA).

\section{$\Delta \mathrm{Vs}$}

The increase of tissue Doppler systolic velocity observed during low dose dobutamine stress $(\Delta \mathrm{Vs})$ is considered an indicator of contractile reserve. The comparison between the three groups showed that $\Delta$ Vs was significantly higher in stunned $(2.1(1.9) \mathrm{cm} / \mathrm{s})$ than in hibernating $(1.2(1.4) \mathrm{cm} / \mathrm{s}$, $\mathrm{p}<0.05)$ or scarred regions $(1.3(1.2) \mathrm{cm} / \mathrm{s}, \mathrm{p}=0.001)$. Table 3 presents tissue Doppler systolic velocities in stunned, hibernating, and scarred myocardium.

\section{DISCUSSION}

\section{Main findings}

Quantitative analysis of myocardial wall motion showed that there is a gradual decline in wall motion velocities between stunned, hibernating, and scarred myocardium, which is more pronounced during low dose dobutamine infusion. However, stunned myocardium had better preserved dobutamine induced contractile reserve, not only in comparison with scar tissue but also in comparison with hibernating myocardium.

\section{Contractile reserve in stunning and hibernation}

In this study, myocardial regions with severe dysfunction assessed by resting cross sectional echocardiography were classified as stunned, hibernating, or scarred, based on the combined scintigraphic assessment of glucose utilisation and perfusion. ${ }^{9-11}$ It has been hypothesised that resting perfusion is initially normal in myocardium affected by chronic coronary artery stenosis, and this state has been referred to as chronic stunning. ${ }^{21}$ When this condition is prolonged over time, animal studies have shown that resting perfusion may eventually become reduced, a state referred to as hibernation. ${ }^{1121}$ Along these lines, it is thought that hibernating myocardium is more damaged on the myocyte level than chronically stunned myocardium. Indeed, biopsies taken from hibernating myocardium during surgical revascularisation have shown a gradual loss of sarcomeres and a nearly absent sarcoplasmic reticulum, with accumulation of glycogen in the spaces previously occupied by the myofilaments. ${ }^{22}{ }^{23}$ Thus, following chronic coronary stenosis,

Table 2 Haemodynamic response during dobutamine infusion

\begin{tabular}{llll}
\hline & Baseline & $\begin{array}{l}\text { Dobutamine } \\
(10 \mu \mathrm{g} / \mathrm{kg} / \mathrm{min})\end{array}$ & $\mathbf{p ~ V a l u e ~}$ \\
\hline Heart rate (beats/min) & $74(12)$ & $83(15)$ & $<0.001$ \\
Systolic blood pressure $(\mathrm{mm} \mathrm{Hg})$ & $125(22)$ & $125(19)$ & 0.960 \\
Diastolic blood pressure $(\mathrm{mm} \mathrm{Hg})$ & $76(11)$ & $73(11)$ & 0.018 \\
Rate-pressure product & $9250(2376)$ & $10377(3017)$ & $<0.001$ \\
Wall motion score index & $2.8(0.5)$ & $2.5(1.0)$ & $<0.001$ \\
\hline Values are mean (SD). & & & \\
\hline
\end{tabular}




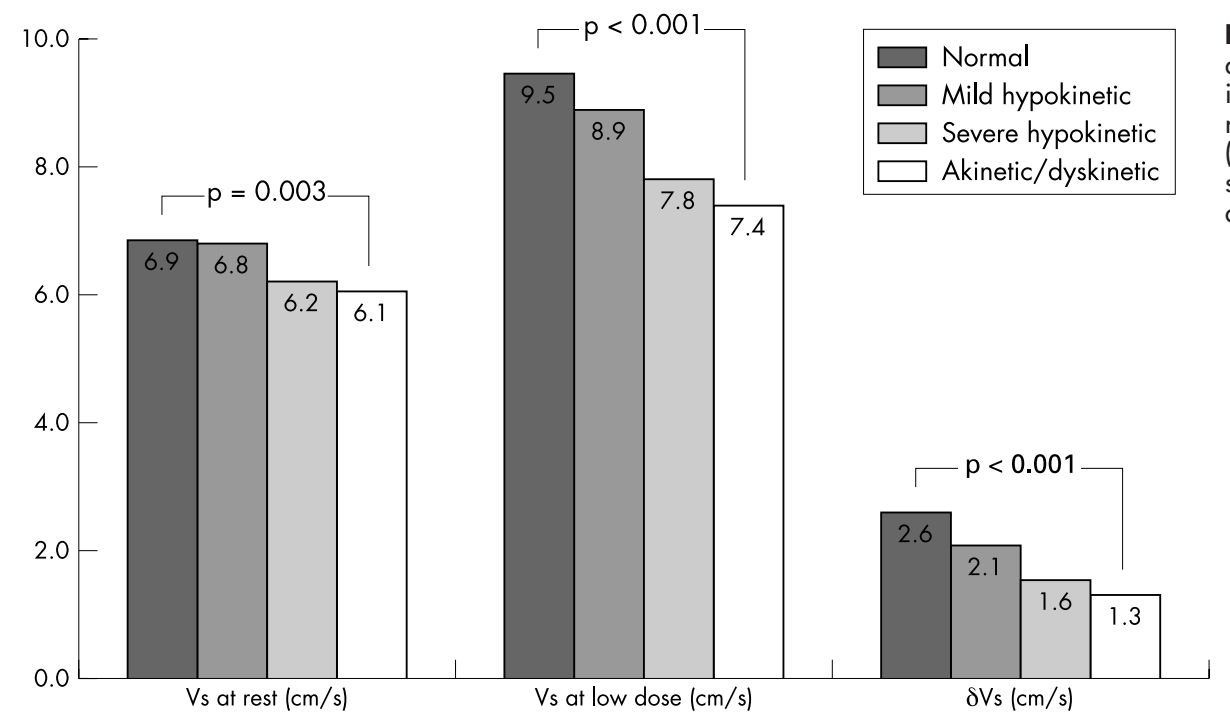

Figure 1 Systolic velocity (Vs) at rest and during low dose dobutamine infusion, and the difference in Vs from rest to low dose dobutamine infusion $(\Delta \mathrm{Vs})$ in normal, mildly hypokinetic, severely hypokinetic, and akinetic/ dyskinetic regions.

stunning may gradually progress to hibernation. ${ }^{11}$ When this condition persists for a long time, irreversible damage may eventually occur, followed by scar formation.

Based on observations on a myocyte level, it is likely that resting contraction and contractile reserve may be more affected (reduced) in hibernating than in stunned myocardium. As cross sectional echocardiography is only based on visual assessment of wall motion, we have used tissue Doppler imaging to provide objective and quantitative information on resting contractile function and contractile reserve (assessed during infusion of low dose dobutamine). Quantitative tissue Doppler imaging done in this way showed a gradual decline in wall motion velocities in stunned, hibernating, and scarred myocardium, reflecting different degrees of ultrastructural damage. In addition, stunned regions had better preserved contractile reserve than either hibernating or scarred regions.

\section{Previous studies}

Our findings are in agreement with previous studies that showed a superior contractile reserve in stunned than in hibernating tissue. Sambuceti and colleagues studied 21 patients one month after they had been given thrombolysis for acute anterior myocardial infarction. ${ }^{22}$ The investigators employed positron emission tomography (using combined assessment of perfusion and glucose utilisation) to differentiate stunned from hibernating myocardium. In stunned myocardium, contractile reserve-assessed by infusion of low dose dobutamine-was frequently intact, whereas hibernating myocardium less often showed contractile reserve. Schinkel and colleagues studied 198 patients with ischaemic cardiomyopathy and also showed that contractile reserve was more commonly present in stunned than in hibernating myocardium. ${ }^{15}$ Our present study confirms and extends these previous findings. These are the first quantitative data on contractile reserve in stunned, hibernating, and scarred myocardium. Tissue Doppler velocities differed significantly in these three situations and may be used to delineate these pathophysiological entities.

\section{Clinical implications}

It is important for clinicians treating patients with ischaemic cardiomyopathy to be able to predict improvement in contractile function after coronary revascularisation. Both hibernating and chronically stunned myocardium (based on the more severe ultrastructural damage) may need a longer time to recover after revascularisation. ${ }^{12}$ Accordingly, the distinction between stunned and hibernating myocardium (and their differentiation from scarred myocardium) may allow the clinician to anticipate the time needed for recovery of function after revascularisation. Quantification of contractile reserve by pulsed wave tissue Doppler imaging may provide an easy, simple, and relatively inexpensive method of acquiring this information. With this in mind, studies in patients undergoing revascularisation are needed.

\section{Limitations}

The study had several limitations. First, no outcome data after myocardial revascularisation were available. Second, pulsed wave tissue Doppler imaging includes the effect of cardiac motion within the thorax, as well as the maintenance of the angle of incidence of the ultrasound beam in relation to the axis of myocardial longitudinal movement; this limitation was partially minimised in our study by using only apical views, where the cardiac apex serves as a fixed reference point. ${ }^{23}$ Finally, tissue Doppler imaging measurements are susceptible to the tethering effect of neighbouring segments. We tried to avoid these effects by considering each of the myocardial walls as a whole region, and by positioning the sample near the mitral annulus, where

Table 3 Pulsed wave tissue Doppler systolic velocities in stunned, hibernating, and scarred segments, at rest and during low dose dobutamine infusion

\begin{tabular}{lllll}
\hline & $\begin{array}{l}\text { Stunning } \\
(\mathbf{n}=132)\end{array}$ & $\begin{array}{l}\text { Hibernation } \\
(\mathbf{n}=\mathbf{2 5})\end{array}$ & $\begin{array}{l}\text { Scarred tissue } \\
(\mathbf{n}=96)\end{array}$ & $\begin{array}{l}\text { p Value } \\
\text { (ANOVA) }\end{array}$ \\
\hline Vs at rest $(\mathrm{cm} / \mathrm{s})$ & $6.3(1.8)$ & $6.6(2.2)$ & $5.5(1.5)$ & 0.001 \\
Vs at low dose $(\mathrm{cm} / \mathrm{s})$ & $8.3(2.6)$ & $7.8(1.5)$ & $6.8(1.9)$ & $<0.001$ \\
$\Delta V_{s}(\mathrm{~cm} / \mathrm{s})$ & $2.1(1.9)$ & $1.2(1.3)$ & $1.3(1.2)$ & 0.001 \\
\hline Values are mean (SD). & & & \\
\hline
\end{tabular}


the vectorial sum of velocities along the longitudinal axis can be measured.

\section{Conclusions}

Quantitative tissue Doppler imaging showed a gradual reduction in wall motion velocities from stunned to hibernating and finally to scarred myocardium. The dobutamine induced contractile reserve assessed by tissue Doppler imaging was greater in stunned regions than in hibernating and scarred myocardium. The gradual reduction in wall motion velocities probably reflects increasing severity of ultrastructural damage in these different pathophysiological entities.

\section{Authors' affiliations \\ M Bountioukos, A F L Schinkel, V Rizzello, B J Krenning, E Biagini, E C Vourvouri, J R T C Roelandt, D Poldermans, Thoraxcenter, Department of Cardiology, Erasmus MC, Rotterdam, Netherlands J J Bax, Department of Cardiology, Leiden University Medical Centre, Leiden, Netherlands \\ R Valkema, Department of Nuclear Medicine, Erasmus MC}

\section{REFERENCES}

1 Rahimtoola SH. From coronary artery disease to heart failure: role of the hibernating myocardium. Am J Cardiol 1995;75:16-22E.

2 Lee KS, Marwick TH, Cook SA, et al. Prognosis of patients with left ventricular dysfunction, with and without viable myocardium after myocardial infarction. Relative efficacy of medical therapy and revascularization. Circulation 1994:90:2687-94.

3 Eitzman D, al-Aouar Z, Kanter HL, et al. Clinical outcome of patients with advanced coronary artery disease after viability studies with positron emission tomography. J Am Coll Cardiol 1992;20:559-65.

4 Bax JJ, Cornel JH, Visser FC, et al. Prediction of improvement of contractile function in patients with ischemic ventricular dysfunction after revascularization by fluorine-18 fluorodeoxyglucose single-photon emission computed tomography. J Am Coll Cardiol 1997;30:377-83.

5 Baumgartner $\mathbf{H}$, Porenta G, Lau YK, et al. Assessment of myocardial viability by dobutamine echocardiography, positron emission tomography and thallium-201 SPECT: correlation with histopathology in explanted hearts. J Am Coll Cardiol 1998;32:1701-8

6 Sciagra R, Bisi G, Santoro GM, et al. Comparison of baseline-nitrate technetium-99m sestamibi with rest-redistribution thallium-201 tomography in detecting viable hibernating myocardium and predicting postrevascularization recovery. J Am Coll Cardiol 1997;30:384-91

7 Senior R, Kaul S, Raval U, et al. Impact of revascularization and myocardial viability determined by nitrate-enhanced Tc-99m sestamibi and Tl-201 imaging on mortality and functional outcome in ischemic cardiomyopathy. J Nucl Cardiol 2002;9:454-62.
8 Bax JJ, Wijns W, Cornel JH, et al. Accuracy of currently available techniques for prediction of functional recovery after revascularization in patients with left ventricular dysfunction due to chronic coronary artery disease: comparison of pooled data. J Am Coll Cardiol 1997;30:1451-60.

9 Rahimtoola SH. A perspective on the three large multicenter randomized clinical trials of coronary bypass surgery for chronic stable angina. Circulation 1985;72:V123-35.

10 Vanoverschelde JL, Wiins W, Depre C, et al. Mechanisms of chronic regional postischemic dysfunction in humans. New insights from the study of noninfarcted collateral-dependent myocardium. Circulation 1993:87:1513-23.

11 Fallavollita JA, Perry BJ, Canty JM. ${ }^{18} \mathrm{~F}$-2-deoxyglucose deposition and regional flow in pigs with chronically dysfunctional myocardium. Evidence for transmural variations in chronic hibernating myocardium. Circulation 1997:95:1900-9.

12 Bax JJ, Visser FC, Poldermans D, et al. Time course of functional recovery of stunned and hibernating segments after surgical revascularization. Circulation 2001;104:1314-18

13 Bax JJ, Visser FC, Poldermans D, et al. Relationship between preoperative viability and postoperative improvement in LVEF and heart failure symptoms. J Nucl Med 2001;42:79-86.

14 Sozzi FB, Poldermans D, Boersma E, et al. Does second harmonic imaging improve left ventricular endocardial border identification at higher heart rates during dobutamine stress echocardiography? J Am Soc Echocardiogr 2000;13:1019-24

15 Schinkel AF, Bax JJ, van Domburg R, et al. Dobutamine-induced contractile reserve in stunned, hibernating, and scarred myocardium in patients with ischemic cardiomyopathy. J Nucl Med 2003;44:127-33.

16 Rambaldi R, Poldermans D, Bax JJ, et al. Doppler tissue velocity sampling improves diagnostic accuracy during dobutamine stress echocardiography for the assessment of viable myocardium in patients with severe left ventricular dysfunction. Eur Heart J 2000;21:1091-8.

17 Nuutila P, Knuuti MJ, Raitakari M, et al. Effect of antilipolysis on heart and skeletal muscle glucose uptake in overnight fasted humans. Am J Physiol 1994:267:E941-6.

18 van Lingen A, Huijgens PC, Visser FC, et al. Performance characteristics of a $511-\mathrm{keV}$ collimator for imaging positron emitters with a standard gammacamera. Eur J Nucl Med 1992;19:315-21.

19 Di Carli M, Sherman T, Khanna S, et al. Myocardial viability in asynergic regions subtended by occluded coronary arteries: relation to the status of collateral flow in patients with chronic coronary artery disease. J Am Coll Cardiol 1994;23:860-8.

20 Schelbert HR. Measurements of myocardial metabolism in patients with ischemic heart disease. Am J Cardiol 1998;82:61-7K.

21 Firoozan S, Wei K, Linka A, et al. A canine model of chronic ischemic cardiomyopathy: characterization of regional flow-function relations. Am J Physiol 1999:276:H446-55.

22 Sambuceti G, Giorgetti A, Corsiglia L, et al. Perfusion-contraction mismatch during inotropic stimulation in hibernating myocardium. J Nucl Med 1998:39:396-402.

23 Garcia MJ, Rodriguez L, Ares M, et al. Differentiation of constrictive pericarditis from restrictive cardiomyopathy: assessment of left ventricular diastolic velocities in longitudinal axis by Doppler tissue imaging. J Am Coll Cardiol 1996:27:108-14

\section{ELECTRONIC PAGES}

\section{Heart Online case reports: www.heartjnl.com}

The follow electronic only articles are published in conjunction with this issue of Heart.

\section{Star in the heart}

K M Krishnamoorthy, J A Tharakan, S R Krishnamanohar In a 50 year old woman, transthoracic echocardiography showed a left atrial mass. Transoesophageal echocardiography delineated its attachment. Additionally, cystic spaces were seen arranged concentrically in the shape of a star in the centre of the tumour. Surgical excision followed. Histopathological examination confirmed myxoma with areas of haemorrhage and necrosis. This case highlights the acoustic property of myxomas in a rare and beautiful manner and emphasises the superior transoesophageal imaging of myxomas.

(Heart 2004;90:e23) www.heartjnl.com/cgi/content/full/90/ $5 / \mathrm{e} 23$

\section{Giant left atrial mass in an asymptomatic patient}

S Lamparter, R Moosdorf, B Maisch

A large atrial myxoma, attached in an atypical location, was identified in the left atrium of a 70 year old patient. Although the tumour occupied a large part of the left atrium the patient remained in sinus rhythm and displayed no symptoms.

(Heart 2004;90:e24) www.heartjnl.com/cgi/content/full/90/ $5 / \mathrm{e} 24$ 